\title{
Bilateral orbital abscesses and cavernous sinus thrombosis in a young patient with Immunoglobulin-A deficiency
}

\author{
A Khanna, D Kapila, H A Saleh
}

Imperial College Healthcare NHS Trust, London, United Kingdom

\section{Background}

While rare in itself, Immunoglobulin-A deficiency (IgAD) is the most common inherited primary immunodeficiency(1), rendering patients susceptible to recurrent gastro-intestinal and respiratory infections, including chronic rhinosinusitis(2); however, no cases of IgAD and acute rhinosinusitis (ARS) or orbital abscesses have been described.

A 15-year-old female presented with one-week history of coryzal symptoms, and three-day history of facial pain, headache, and pyrexia, bilateral proptosis and peri-orbital cellulitis, worse on the right. Computed tomography (CT) and magnetic resonance imaging (MRI) showed pansinusitus, raised intracranial pressure and cavernous sinus thrombosis. Endoscopic sinus surgery (ESS) revealed pus in the right sphenoid sinus.
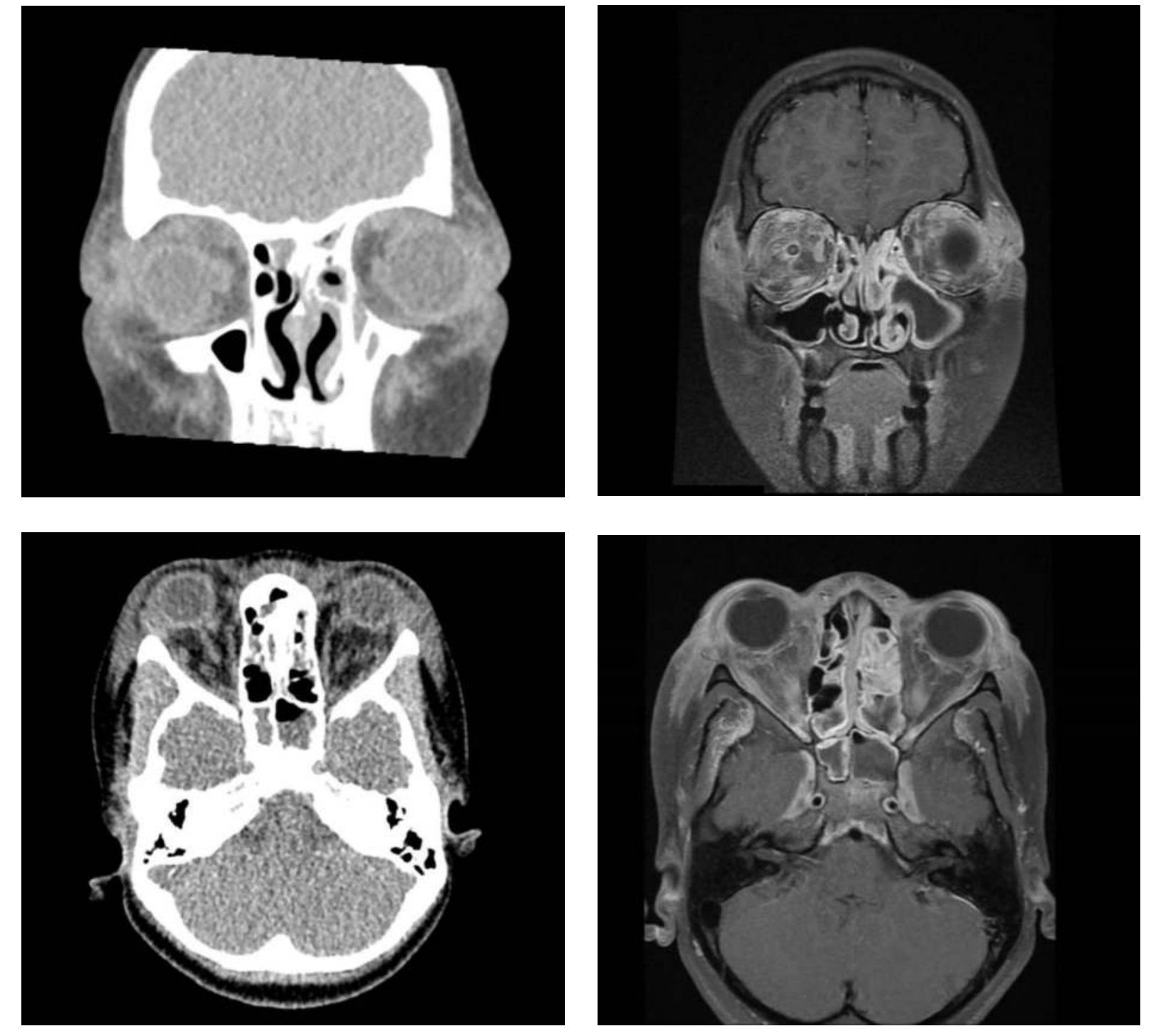

Despite systemic antimicrobial therapy, the patient developed opthalmoplegia, diplopia and bilateral abducens nerve palsies. Magnetic resonance imaging revealed intra- and extra-conal orbital empyemas
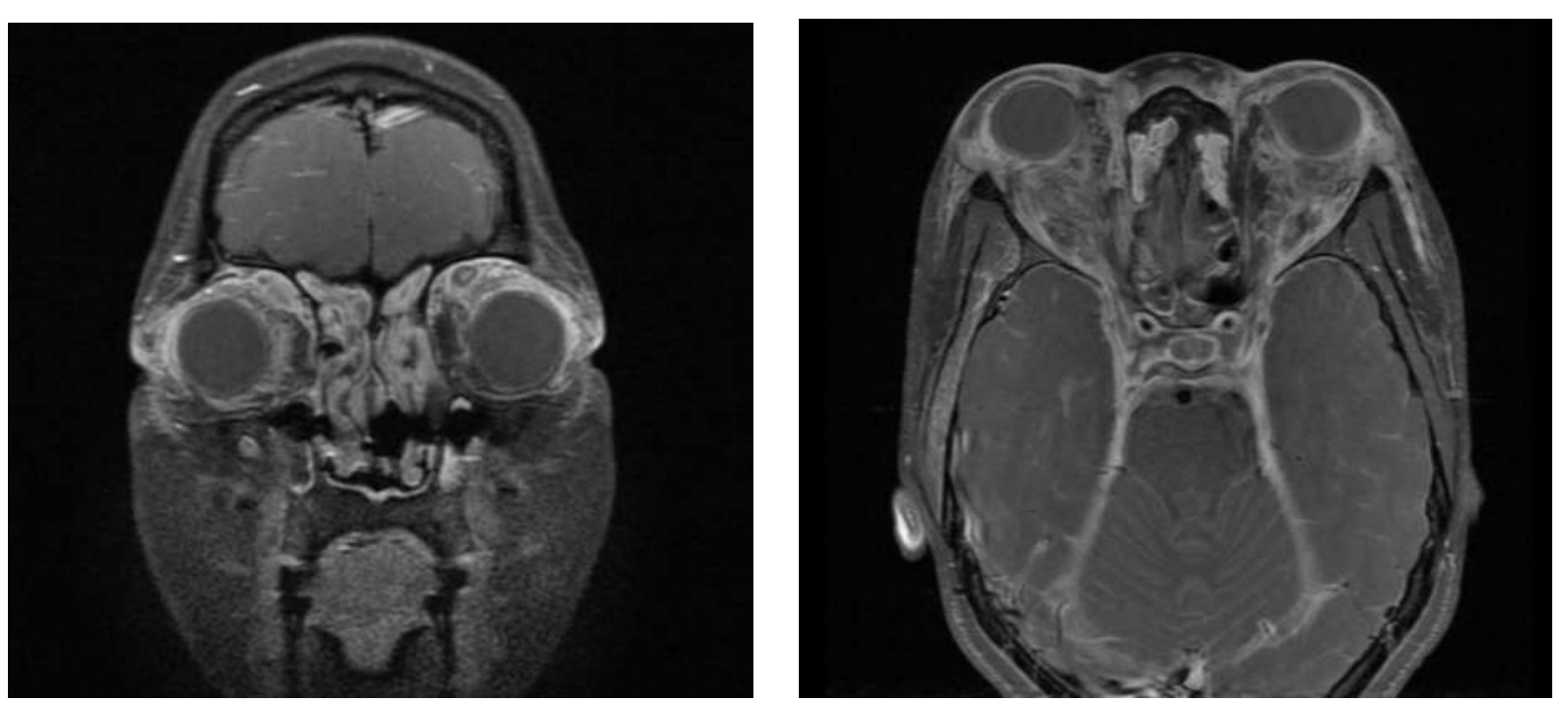

\section{Method}

Bilateral endoscopic medial orbital wall and partial inferior wall orbital decompression was performed along with complete ESS. The patient was anti-coagulated with haematology and paediatric neurology advice.

\section{Results}

Further investigation revealed IgAD which was managed appropriately in the Combined Paediatric Immunodeficiency Clinic.

A further episode of unilateral right periorbital cellulitis two months later was managed conservatively.
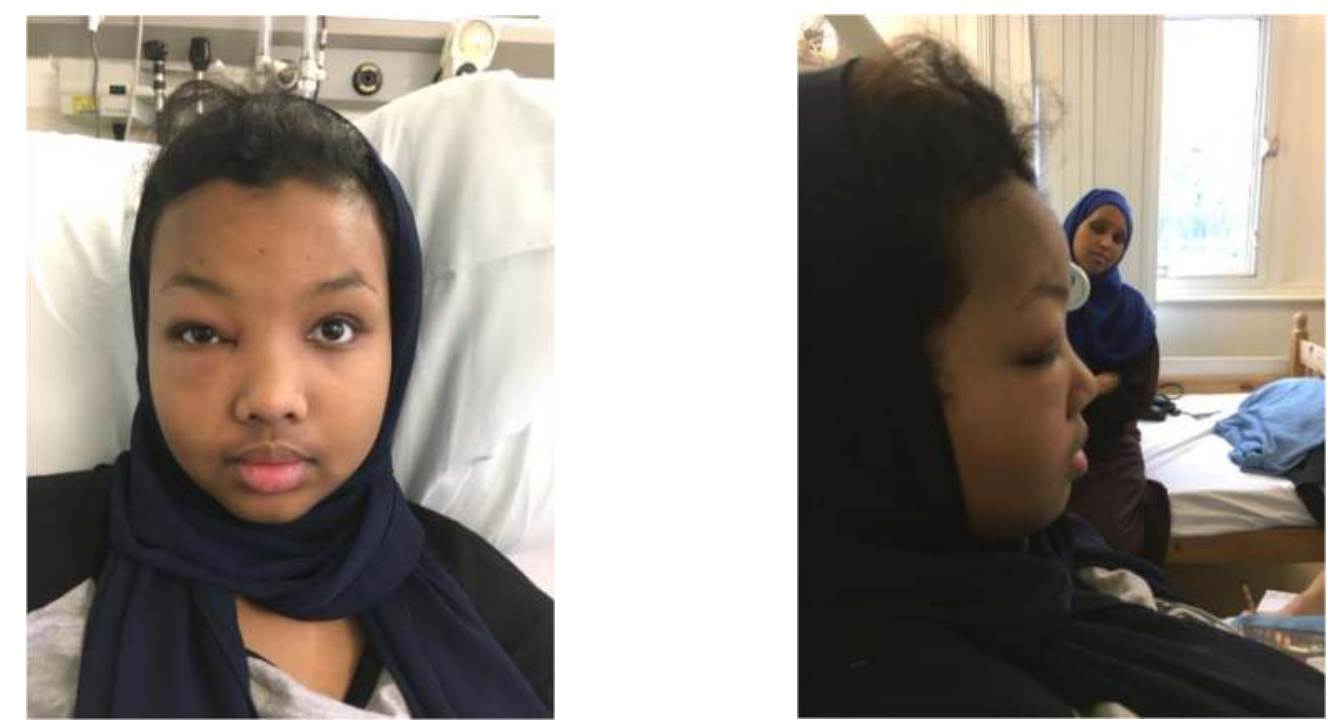

At 12-month follow-up all symptoms had resolved.
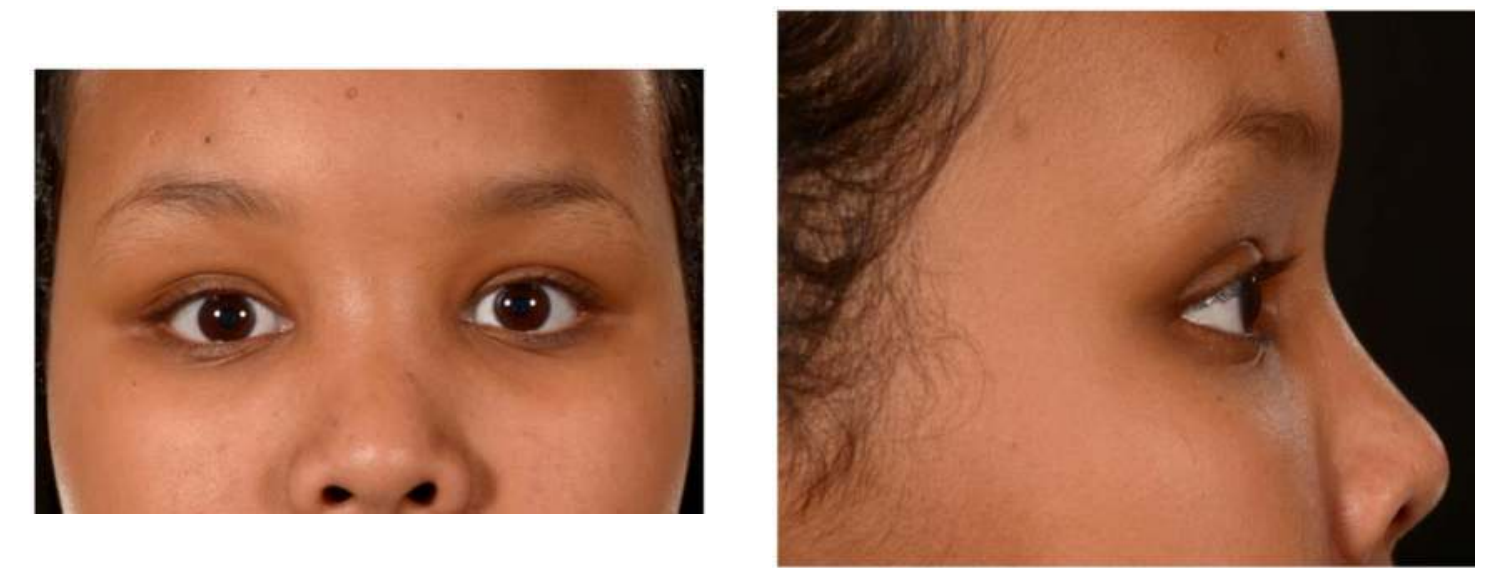

\section{Conclusion}

Immune deficiencies should be considered in complicated cases of sinusitis. Prompt diagnosis of immune deficiency is imperative to improve clinical outcome. A multi-disciplinary team approach is essential in complex cases.

\section{Reference}

1. Stiehm ER. The four most common pediatric immunodeficiencies. J Immunotoxicol. 2008 Apr;5(2):22734.

2. Schwitzguébel AJ, Jandus P, Lacroix JS, Seebach JD, Harr T. Immunoglobulin deficiency in patients with chronic rhinosinusitis: Systematic review of the literature and metaanalysis. J Allergy Clin Immunol. 2015;136(6):1523-1531. 